\title{
Recommendations of the Polish Society of Gynaecologists and Obstetricians for removal of the uterus by vaginal, laparoscopic and abdominal routes
}

\author{
Rafal Stojko ${ }^{1,2}{ }^{-}$, Andrzej Malinowski ${ }^{3}$, Wlodzimierz Baranowski ${ }^{4}$, Marcin Misiek ${ }^{5}$, \\ Ewa Winkowska' ${ }^{1}$, Michal Pomorski ${ }^{6}\left(\mathbb{D}\right.$, Mariusz Zimmer ${ }^{6}(0)$ \\ ${ }^{1}$ Department of Obstetrics and Gynecology with the Subdivision of Oncological Gynecology, \\ Brothers Hospitallers of Saint John of God Hospital in Katowice, Poland \\ ${ }^{2}$ Chair and Clinical Department of Gyneacology, Obstetrics and Oncological Gyneacology, Medical University of Silesia, Katowice, Poland \\ ${ }^{3}$ Department of Operative Gynecology, Endoscopy and Gynecologic Oncology, \\ Polish Mother's Memorial Hospital Research Institute, Lodz, Poland \\ ${ }^{4}$ Gynecology and Oncological Gynecology Clinic of the Military Institute of Medicine, Warsaw, Poland \\ ${ }^{5}$ Clinical Department of Gynaecological Oncology, Świętokrzyskie Cancer Centre, Kielce, Poland \\ ${ }^{6} 2^{\text {nd }}$ Department of Gynecology and Obstetrics, Wroclaw Medical University, Wroclaw, Poland
}

\begin{abstract}
The recommendations represent the current procedure, which may be modified and changed where justified, after a thorough analysis of the given clinical situation, which may be the basis for their modification and updating in the future.
\end{abstract}

Key words: hysterectomy; transvaginal; laparoscopic

Ginekologia Polska 2020; 91, 6: 352-361

\section{Objectives}

The purpose of these recommendations is to develop indications for the removal of the uterus by vaginal, laparoscopic and abdominal routes. The guidelines are based on the latest literature reports and the experience of the authors.

\section{INTRODUCTION}

A hysterectomy is one of the most frequently performed gynecological procedures and one of the most frequent elective operations in the world. Uterine removal can be performed in several ways: transabdominal (TAH/AH), laparoscopic (LH), vaginal (TVH/VH) and robot access [1-3]. Indications for a hysterectomy for non-cancerous reasons are most often: symptomatic uterine myomas, abnormal bleeding from the reproductive tract, endometriosis or genital depression/exclusion [4, 5]. According to ACOG, transvaginal removal of the uterus is the safest and most cost-effective method of hysterectomy for non-cancerous indications [1]. Laparoscopy also has many advantages, such as image mag- nification providing better visualization of anatomical structures and identification of the disease outside the uterus. It also allows better access to retroperitoneal spaces thanks to pneumodissection [6, 7]. Both types of minimally invasive surgery $(\mathrm{VH} ; \mathrm{LH})$ compared to transabdominal surgery is associated with shorter surgery time, less blood loss, fewer transfusions, shorter hospital stays, fewer used painkillers, faster return of intestinal work, shorter recovery time and faster return to normal daily life and work activities $[5,8]$. All these factors make these methods of uterine removal much more beneficial also in socio-economic terms [9].

Despite these undisputed advantages, both in Poland and worldwide, the dominant way of performing a hysterectomy is still via laparotomy. This may be due to the lack of appropriate emphasis on minimally invasive operations during the training of gynaecology and obstetrics specialists, the lack of clear guidelines enabling appropriate qualification for this type of procedures, the habit of operators or finally the lack of knowledge of patients about operating techniques [10, 11]. 
According to NHF (National Health Fund) data, 35,025 hysterectomies were performed in Poland in 2009, of which as many as $22,526(64 \%)$ were performed via the abdominal tract (TAH), of which $10148(28 \%)$ - supracervical amputations (TASH), and only $1740(5 \%)$ - vaginal tract (TVH), and 611 (1.8\%) - laparoscopic (LASH/TLH). In 2013 the percentage distribution of methods of performing hysterectomies practically did not change: abdominal surgery - 16,443 (53\%), including TASH - 11,655 (38\%), TVH 1292 (4\%), LASH/TLH $-1,582(5 \%)$. As we can see, minimally invasive surgeries (TVH, LASH/TLH), despite their advantage over transabdominal surgeries, are still performed in Poland in a significant minority of cases (less than 10\% hysterectomy).

\section{Indications for removal of the uterus for non- oncological reasons}

There are a number of indications for uterine excision for non-oncological reasons. The choice of an appropriate surgical method should depend on the type of condition (Tab. 1).

One of the most common indications for hysterectomy are abnormal or not responding to hormonal treatment uterine bleeding. The basis for the diagnosis of the disease is exclusion of uterine cavity and endometrium pathology. Prior to surgical treatment, patients with abnormal uterine bleeding should undergo laboratory diagnosis (evaluation of blood morphology and biochemical parameters), imaging (e.g.: ultrasound examination and optional hysteroscopy) and fractional abrasion from the canal and uterine cavity. In case of normal laboratory results and non-oncological histopathological examination, hormone therapy (progestogens, $\mathrm{E}+\mathrm{P}$ or tibolone) is indicated for at least three months. In the absence of improvement after pharmacological treatment, the patient is a candidate for a hysterectomy, of course depending on age and procreation plans.

The second and most common reason for the patient's qualification for a hysterectomy are myomas. Most

Table 1. Indications for uterine removal for non-oncological reasons

\begin{tabular}{|l|l|l|l|}
\hline & VH & LH & AH \\
\hline Functional uterine bleeding & 1 & 2 & \\
\hline Intrauterine adenomyosis/Adenomyosis & 1 & 2 & \\
\hline Uterine fibroids: uterus size $<12 \mathrm{hbd}$ & 1 & & \\
\hline uterus size $14-16 \mathrm{hbd}$ & $2,1^{*}$ & 1 & 3 \\
\hline uterus size $17-20 \mathrm{hbd}$ & $1^{*}$ & 1 & 2 \\
\hline uterus size $>$ 20-24 hbd & $1^{*}$ & & 1 \\
\hline Recurring endometrial polyps & 1 & 2 & \\
\hline Endometrial hyperplasia (with or without atypia) & 1 & 2 & \\
\hline
\end{tabular}

1 - first choice; 2 - second choice; 3 - third choice; ${ }^{*}$ for experienced gynaecologists women with uterine myomas complain of abnormal, heavy and irregular uterine bleeding (30\%) and abdominal pain (39\%). In asymptomatic patients, the diagnosis is based on a two-handed gynecological examination and ultrasound diagnostics. Sometimes it is also helpful in the assessment of myomas to extend the diagnosis by magnetic resonance imaging or, less frequently, computed tomography.

Patients with asymptomatic uterine myomas do not require any treatment but only systematic gynaecological control at six-month intervals. Indications for surgery due to uterine myomas are ineffective non-operative treatment of myomas and recurrence of symptoms after non-operative treatment.

When choosing the surgical technique for a hysterectomy, the size and volume of the uterus is important and not the number of myomas. According to the ACOG recommendation of 2009 in patients qualified for a hysterectomy, the size of the uterus not exceeding 12 weeks of pregnancy (about 280-360 g) is an optimal indication for a transvaginal hysterectomy [12]. For larger sizes (volumes) of the uterus, the laparoscopic route $(\mathrm{LH})$ is a better choice. The last option (difficult or risky laparoscopic technique, low operator experience) should be considered for an abdominal hysterectomy.

There is no doubt that as experience is gained in vaginal techniques, the size of the uterus over 12 weeks of pregnancy and the volume over $300 \mathrm{~cm}^{3}$ is not contraindicated for a vaginal hysterectomy. As proved by Cho HY et al. [13], transvaginal method is safe even if the uterine weight exceeds $500 \mathrm{~g}$ - the most important in choosing this surgical route is the experience of the operator. In the case of large uteruses, an important factor in the choice of the vaginal route is its vaginal "availability", i.e. such factors as uterine size, mobility (possible lowering), vaginal size and the angle between the cervix and the corpus. Vaginal width can be assessed by physical examination - the wider and shorter the vagina, the easier access to the uterus will be and the surgical field will be more visible [11, 13, 14]. The angle between the cervix and the corpus can be assessed during ultrasound (Fig. 1). If the angle between the lateral boundaries of the uterus is greater than $140^{\circ}$, it facilitates the gynaecologist's vaginal excision. An angle of less than $90^{\circ}$ makes vaginal access to the uterine vessels and the uterine shaft difficult or even impossible.

The location of the myomas must also be considered when accessing the way of excision of the uterus in case of myomas. In the case of large size myomas (more than $6 \mathrm{~cm}$ in diameter) with a subcutaneous location on the anterior wall of the uterus shaft, the vaginal hysterectomy may be, for less experienced operators, very difficult or even impossible to perform due to the low downward retractability of the uterus (blocking the uterus on the pubic bones). It is worth noting that the method of choice in the surgical treatment of submucous fibroids is surgical hysteroscopy [15]. 


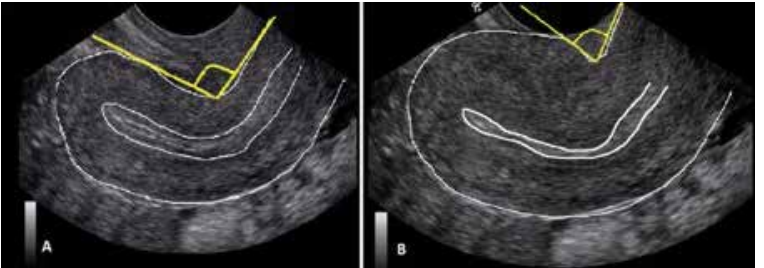

Figure 1. Relationship between the size of the uterus and the angle of the lateral boundaries of the corpus uteri and the cervix A - uterus of correct size; $B$ - enlarged uterus

A particular situation and indication for uterine excision is adenomyosis. Adenomyosis, i.e. endometrial adenomyosis, is characterized by the presence of endometrial tissue and bedding outside the uterine mucosa. Often the only and final basis for diagnosis and differentiation from abnormal uterine bleeding is an in-depth histopathological examination of the excised uterus. The adenomyotically altered uteruses are often enlarged and sometimes heterogeneous. In the case of significantly enlarged uteruses, an experienced gynaecologist can perform a vaginal hysterectomy, using appropriate techniques of morcellation of this organ.

Another indication for a hysterectomy is painful menstruation, which often accompanies adenomyosis or endometriosis of the smaller pelvic organs.

With ineffective analgesic and hormonal treatment, uterine excision seems to be the only effective method.

There is also a group of patients suffering from chronic pelvic pain.

In a significant percentage of cases the pain is associated with severe peritoneal endometriosis (usually with the presence of intraperitoneal adhesions) or with the presence of deeply infiltrating endometriosis. Although in these situations uterine excision seems to be controversial, after using all available pain treatment methods, hysterectomies give hope for improvement of ailments and well-being.

An important indication for uterine excision is the overgrowth of the uterine mucosa (endometrium). The therapeutic management depends on the histopathological outcome of the scrawls from the canal and uterine cavity. Uterine excision seems unjustified in cases of simple proliferation due to lack of evidence of endometrial neoplasia. Patients with complex endometrial proliferation without atypia should initially undergo a three-month hormone therapy containing progestogens, and then have the canal and uterine cavity fractionated abrasion performed again. The initial hormone treatment in this case is justified due to the low risk of disease progression. If a repeated histopathological examination shows endometrial proliferation again, the uterus should be excised. In the case of complex endometrial proliferation with the atypia, treatment of choice is the operation of uterine removal.

Additional attention is required for patients with severe dysplasia or cervical cancer in situ. Routine hysterectomy due to dysplasia or pre-invasive cervical cancer is not recommended. The choice of treatment should consider the severity of the disease, the age of the patient and maternal plans. For those patients who do not want to have more children or those with a relapse or after an unfortunate excision of the lesion, it is best to remove the uterus. Early cervical cancer of grade la1 (without occupying vascular and lymphatic space) requires nothing more than a simple hysterectomy. The choice of laparoscopic or abdominal route is not recommended if no pathology is found in the appendages.

Rare indications for uterine excision are also recurrent stem and cervical polyps.

Table 2. shows general and indicative "tactical" assumptions in the choice of the path to uterine excision. They may

Table 2. Choice of hysterectomy for non-oncological reasons

\begin{tabular}{|c|c|}
\hline \multicolumn{2}{|l|}{ Choice of hysterectomy method } \\
\hline \multicolumn{2}{|l|}{ Vaginal hysterectomy (VH) 70-80\% } \\
\hline Abdominal hysterectomy (AH) $5 \%$ & Laparoscopic hysterectomy (LH) $15-25 \%$ \\
\hline $\begin{array}{l}\text { - lack of experience in vaginal and laparoscopic operations } \\
\text { - coexistence of non-gynecological pathology* } \\
\text { - large uterus over } 20-24 \text { weeks of pregnancy* } \\
\text { - pathology of the appendages* } \\
\text { - extensive/massive intraperitoneal adhesions - status post } \\
\text { numerous abdominal surgeries* }\end{array}$ & $\begin{array}{l}\text { - } \text { necessity of abdominal inspection } \\
\text { - coexistence of non-gynecological pathology* } \\
\text { - large uterus over } 16 \text { weeks of pregnancy* } \\
\text { - the need to remove appendages* } \\
\text { - pathology of the appendages* } \\
\text { - } \text { advanced endometriosis, deeply infiltrating endometriosis } \\
\text { - } \text { extensive/massive intraperitoneal adhesions (PID, status post abdominal } \\
\text { surgery, abdominal pain) } \\
\text { - narrow vagina } \\
\text { - nullipara/no vaginal delivery* } \\
\text { - immobile uterus } \\
\text { - no access to the cervix (status post cervical amputation/very small cervix)* } \\
\text { - lack of experience in vaginal operations }\end{array}$ \\
\hline
\end{tabular}

* - commentary in the text of the paper 
be a guideline for gynaecologists training in vaginal and laparoscopic hysterectomy techniques. According to the recommendations of most gynaecological scientific societies and our own experience, a vaginal hysterectomy is possible in $70-80 \%$ of cases of indications for uterine excision. An abdominal hysterectomy (laparotomy) should be performed only in about $5 \%$ of cases of indications for uterine excision for non-oncological reasons. The remaining 15-25\% of hysterectomies should be performed laparoscopically. The laparoscopic technique should be used when vaginal access is difficult and the operator's experience is insufficient in the case of nullipara, large uterus (size 16 weeks is usually the upper limit of TVH) when uterine mobilization is insufficient [16].

To meet such guidelines, in addition to adequate training in vaginal and laparoscopic hysterectomies, it is necessary to completely change the mentality during the qualification process for the decision on the method of uterine excision. If the decision has aready been made to remove the uterus, the vaginal route should be considered first. The alternative way to remove the uterus should only result from contraindications or serious difficulties in the vaginal tract.

So, when is the appropriate time for a laparascopy or laparotomy? A few remarks from the commentary to Table 2. The coexistence of non-gynecological pathology may be an indication for laparotomy or laparoscopy. The decisive factor in this case will be the experience of the surgeon who will operate such a pathology.

Myomas is almost a classic indication for a vaginal hysterectomy. For an experienced vaginal surgeon, the size of the uterus even above $1 \mathrm{~kg}$ will also not be a contraindication for such surgery. However, in the initial period of a vaginal hysterectomy, a laparoscopic route should be considered for uterus sizes over 16 weeks of pregnancy, and a laparotomy should be considered for uteruses over 20-24 weeks of pregnancy.

During a vaginal hysterectomy most often the fallopian tubes and ovaries can be removed without major problems - this can be done in $70-85 \%$ of cases. The decision to remove the ovaries and/or fallopian tubes is independent of the method of hysterectomy and is not contraindicated for transvaginal removal $[11,17]$. In the case of absolute necessity to remove adnexa during a hysterectomy (e.g. endometrial carcinoma G1), the route or laparoscopic assistance should be considered.

The coexisting pathology of appendages is an indication for laparoscopy or laparotomy. The choice of surgical route depends on many factors, including the nature of the lesion (solid, fluid, etc.), vascularisation, size, laparoscopic experience, etc. If the operator has doubts about the transvaginal hysterectomy due to ovarian or fallopian tube diseases, deep endometriosis or adhesions, it is acceptable to visualize the pelvis smaller with a laparoscopic probe. This will allow the anatomy to be assessed and a final decision to be made on how to operate $[10,12]$.

A difficult decision to choose the route of uterine excision will depend on a patient after many abdominal surgeries, especially surgical, intestinal (often with a history of postoperative bowel obstruction). In this case extensive intraperitoneal adhesions, intraoperative intestinal damage and postoperative intestinal obstruction can be expected. Both laparoscopy and laparotomy are exposed to such complications. Sometimes in such cases it is worthwhile to choose the vaginal route bearing in mind that the operation takes place largely outside the peritoneum, the adhesions usually cover the bottom of the uterus shank, which means that we do not have to remove the adhesions to the same extent as during laparoscopy or laparotomy (operations from the top, i.e. before we get to the uterus we have to remove the adhesions and intestines), so the risk of postoperative obstruction is much lower.

For an experienced vaginal surgeon, a situation of lack of birth by vaginal route (or nullipara) and lack of access to the cervix (e.g. very small cervix, condition after cervical amputation) are not contraindications for vaginal excision. In most cases, a vaginal hysterectomy can be performed in women who have not given birth or have undergone C-section [18, 19]. According to Tohic AL et al. [20], even in $92 \%$ of women in this group, this route of uterine removal may be successful. It should be noted that the lack of access to the cervix (e.g. the very small cervix, the condition after cervical amputation) will also be a major obstacle for laparoscopic hysterectomy, as the insertion of a collar-type uterine manipulator onto the cervix may be difficult or impossible.

\section{Technique of excision of the uterus through the vaginal tract (TVH)}

The patient is placed in a lithotomy position for a transvaginal hysterectomy. Attention should be paid to the preparation staff to ensure good access to the surgical field - the buttocks of the patient should be placed slightly beyond the edge of the operating table, preferably the lower limbs should be bent in the hip joints at least 90 degrees. This is not always possible, especially in elderly patients or patients with orthopaedical diseases of this area of the body. When arranging the patient, excessive bending and abduction of the thighs should be avoided, as this may lead to temporary or permanent nerve damage caused by such positioning - especially pressure on the lateral surfaces of the lower leg should be avoided due to the risk of fibular nerve damage.

Once the surgical field is decontaminated and draped, the patient is obligatorily catheterized; if left during surgery, the catheter is usually placed on the lower surface of the 
patient's left thigh. The displacement of the catheter over the groin should be avoided - this often leads to blockage of urinary outflow and lack of control over diuresis. After anaesthesia, an examination of the patient is carried out to check the degree of uterine prolapse, vaginal width and the presence of possible changes in the pelvis minor other than myomas.

After the insertion of bivalve speculum (most often Kallmorgen speculum, less frequently Dever or Haney's retractors) and after the cervix is visible, the vaginal part of the cervix is fastened with bullet forceps. In the case of significant anatomical damage to the cervix (extensive cracks up to the vaults), the bullet forceps should be fastened so that the shape of the cervix remains its anatomical form as much as possible. In patients with prior cervical amputation, the cervical stump should be grasped in such way that the vaginal vauts can be dissected and access to the fastening point of the bullet forceps be gained without the risk of the walls of the bladder being drawn into the bullet forceps and then into the operating field. In these patients, it is recommended to inject the vaginal wall with a saline solution or ready-made solutions of $1 \%$ or $2 \%$ lidocaine or $0.5 \%$ bupivacaine with an adrenaline solution titrated to $1: 200,000$. In practice, preparation of the solution for hydro-dissection consists of adding to $20 \mathrm{~mL}$ of local anesthetic solution (lidocaine, bupivacaine) or $20 \mathrm{~mL}$ of saline solution 4 drops (about 200 microliters) of $0.1 \%$ adrenaline solution. Please note that the maximum amount of lidocaine should not exceed $7 \mathrm{mg} / \mathrm{kg}$ or a total of $500 \mathrm{mg}$ for a healthy adult and the amount of bupivacaine should not exceed $225 \mathrm{mg}$. Practically during transvaginal hysterectomy the total volume of hydro-dissection solution does not exceed $10 \mathrm{~mL}$. This procedure significantly facilitates the preparation of the space between the bladder and cervical stump. The injection procedure described above should not be used routinely in each patient as it may significantly change (shift) the site of the original vaginal wall incision. In order to determine the position of the bladder, a manoeuvre consisting in slight overhead pushing of the cervix may be performed - the position of the bladder wall is indicated by a fold of the vaginal wall (bladder reflection).

In the case of non-oncological transvaginal hysterectomy, it begins at an incision of the vaginal wall in the immediate vicinity of the cervix (maximum $1.5-2 \mathrm{~cm}$ from the external cervical os) with maintaining the peri-cervical ring structures (equivalent to the transabdominal uterine excision by Aldridge's method). In patients with oncological conditions (e.g. ca endometrial low grade, cervical dysplasia) the distance of the vaginal wall incision from the external cervical os depends on oncological indications and is the responsibility of the operator. If a significant vaginal fragment has to be excised (e.g. extensive severe dysplasia), the risk of bladder damage during the preparation from both the vaginal wall and cervical side should be kept in mind.

The first incision of the vaginal wall at the cervix should be made perpendicular to the axis of the cervix up to the cervical stroma - at the right depth of the incision, the vaginal wall will usually separate from the cervix by itself. The vaginal wall incisions are usually made with monopolar tools, in which we use a coagulation module or, less frequently, with a scalpel. Vaginal walls are usually incised in a circular manner, some operators in patients with a large uterus make an incision of the posterior vaginal wall in which the sharp end of the letter $V$ reaches the posterior vaginal vault (Benenden incision) to enlarge the access field to the uterus. A very helpful tool at this stage of the operation is the Breisky retractors.

After cutting the vaginal walls and gaining access to descending bunches of branches of uterine vessels, usually with power tools closing blood vessels or sutures, cervical vessels (branches of descending uterine vessels) should be ligated. Modern medical technologies offer power tools for closing large (up to $7 \mathrm{~mm}$ in diameter) blood vessels (vascular sealing) and have significantly influenced surgical possibilities, even contributing to changes in surgical procedures.

In the next stage of surgery, we gently move the front and back vaginal wall upwards, gaining access to the front and back fold respectively. An important practical tip is to find the right layer of preparation, both in the anterior and posterior compartment - then it is an avascular space and we practically do not observe bleeding. Of course, in the case of a large uterus, the preparation of peri-cervical tissues is multistage, but as a rule we should strive to open the posterior peritoneal fold (pouch of Douglas) first. It is helpful at this stage to find and identify the sacro-uterine ligaments - between them the peritoneum of Douglas's fold can be easily identified and after grasping it with tweezers, cut with scissors. After opening the peritoneal cavity of the posterior fold, we often observe the leakage of a small amount of serous fluid and very clearly tension the sacro-uterine ligaments. After inserting the Breisky retractor into the posterior peritoneal cavity, easily under visual control, we coagulate/split and cut the sacro-uterine ligaments. This manoeuvre gives a very good view and access to the cardinal uterine ligament, which after coagulation/puncture in its lower part we cut off. At this stage of the operation we obtain good uterine mobility that enables the next steps of the operation. The opening of the anterior peritoneal fold (vesico-uterine pouch) is a more difficult stage than the opening of the posterior fold because the parietal peritoneum adheres, sometimes very closely, to the uterus. It is helpful to lift the parietal peritoneum with surgical tweezers and cut under 
visual control. Other manoeuvres (e.g. blunt-finger/gasket preparation) are most often ineffective and contribute to non-physiological peritoneal detachment, the creation of a large dead space and greater postoperative pain. Bleeding from the posterior vaginal wall cuff is quite common - the bleeding is controlled either by cauterisation or by placing a continuous suture (1-0) on the vaginal edge. After gaining access to the anterior wall of the uterus, we continue coagulation/ pricking and cutting off the lateral perimetrium up to the height of the tray of the round ligament of uterus and proper ovarian ligament. All these structures are coagulated/ pricked and cut off in turn. Operators using the traditional technique with vessel pricking stitch the peduncle of the cardinal uterus ligament to the peduncle of the sacro-uterine ligament. In the power tool technique, both these stumps are separate structures.

If the patient qualifies to cut out the fallopian tubes, this can be done at this stage of the operation by finding the abdominal os of the fallopian tube, grasping this part of the fallopian tube with the window forceps and by successively coagulating the mesentery of the fallopian tube and cutting off as close to the fallopian tube as possible, mobilising this organ up to its intramural. This allows removal of the fallopian tube in one tissue block with uterus. In the case of a tight operating field (large uterus) this can be done after the removal of the uterus from the vagina - then it is worthwhile to seal the stump of the appendages with ticks or sutures in order to find them later in the operating field and pull them downwards. In this way it is also possible to remove the pathology of the ovary and, depending on the indications, enucleate the cyst out or remove the ovary(s).

In the case of very large uteruses, a slightly different preparation may be used, involving the maximum release of the cervix (under the given conditions) and then cutting off the cervix. This gives access to the uterus corpus, which becomes much more mobile under these conditions. We continue the preparation of the lateral perimetrium, on the principle of turning like a ball, of the uterine corpus, sometimes changing sides several times (right and left). It is recommended to use power tools to close the vessels instead of the traditional technique of grasping and pricking individual anatomical structures with ticks, as this significantly reduces postoperative pain (less use of analgesics) and reduces tissue reaction, causing faster tissue healing.

In the case of large uteruses, it is necessary to reduce the size of the surgical preparation. Several morcellation techniques can be used. The most common technique is the so-called "coring" technique, which consists in conical incision of the corpus until the size of the uterus can be removed by the vagina. This method is most recommended for safety reasons (protection of the bladder and rectum) and lack of contact of myometrium/endometrium with the peritoneal cavity. In the case of large anatomical deformities of the uterus, usually because of myomas, morcellation is also used but dictated by anatomical conditions. It is worth thinking, in the case of myomas, about intraoperative exfoliation of myomas as a method to reduce the size of the uterus. It also happens that the myoma(s) are left unintentionally in the peritoneal cavity - this phenomenon is observed in the case of myomas which are scattered, detached during pulling the uterus down and "dying" from the operator's field of view in the intestinal loops.

After obtaining and checking the haemostasis, we begin to close the peritoneum and vaginal stump. For this purpose, Kocher's forceps should be used to grasp the stumps of the sacro-uterine ligaments and the front vaginal wall in the midline. While the peritoneum of the back vaginal wall is usually well visible and easy to grasp, peritoneum of the vesico-uterine pouch needs to be found - sometimes its edge is quite deep in the pelvis minor. The vaginal stump is usually stitched transversely by means of a continuous stitch, ensuring that the anterior and posterior peritoneal wall suture line is gripped - this reduces the volume of the so-called dead space, thus reducing the risk of postoperative adhesions or hematoma.

In some centres, the suture of vaginal walls after surgery is used longitudinally, in the sagittal dimension - it is not supposed to change the length of this organ, although the literature data do not prove the rightness of such an approach.

In patients with prior lowering of the uterus and posterior vaginal wall it is worth using McCall's external sutures, which significantly restore proper anatomical conditions and reduce the risk of vaginal stump prolapse in the future.

In patients with no pelvic floor static disorder, no prophylactic surgery is recommended, based on the assumption that correctly grasped and sewn together with the anterior vaginal wall, the sacro-uterine ligaments and the paracervical ring preserved during the vaginal hysterectomy is sufficient to protect the pelvic floor static after this procedure. After cleaning and disinfecting the surgical field, it is recommended to place the seton in the vagina and maintain it together with the Foley catheter until the next day. The patient is usually discharged home on the first, less frequently on the second postoperative day. Postoperative control is recommended 4-6 weeks after the surgery - if the surgical suture is still present, we should gently, usually with tweezers, remove fragments of this material as it no longer has any mechanical functions but is only an irritating element and may contribute to the production of bleeding postoperative granulation tissue. The next control is recommended about 6 months after the operation and is intended, in addition to a routine gynaecological examination, for urogynecological evaluation (possible disorders of miction or pelvic floor static disorders). 
Additional notes:

1. Assessment of pelvic floor static disorders and possible prevention of POP.

During transvaginal hysterectomies, the posterior vaginal wall statics should be routinely assessed, because in the final stage of transvaginal excision surgery the defects of the posterior, central and anterior compartments can be relatively easily and effectively provided. The most used method is McCall's culdoplasty procedure. In case of significant prolapse of the posterior vaginal wall $(P O P Q>2)$ it is recommended to put 2-3 McCall's internal and external sutures, according to the technique described by this operator. The number of McCall's external sutures depends on the degree of prolapse and anatomical conditions of the operated person (usually 1 or 2 sutures). McCall's external sutures are tied only after the vaginal walls are sewn. This surgical technique carries a slight risk of ureter displacement and disturbance of their function (kinking) - therefore, caution should be exercised both in the number of sutures and their location, especially in the cephalad section. Some authors recommend intraoperative cystoscopic control of ureter function (urine jet) - however, this is not an obligatory procedure, especially when fixing small defects in the posterior compartment. In the case of lowering of the anterior vaginal wall, before stitching the cuff, it is also possible to perform a native plastic surgery of the anterior vaginal wall (various surgical techniques). Synthetic materials (mesh) are not recommended due to the high risk of erosion of the prosthetic material.

2. In the light of the available literature data, it is not recommended to routinely use any prophylactic procedures for POPs - patients without any preoperative and during the procedure of pelvic floor static disorders do not require prophylactic POP procedures.

3. Difficulties in opening the anterior and posterior recesses.

Sometimes opening the rear and front recess can be a real challenge for the operator. Most often it is related to past Caesarean section(s) (anterior recess) or past pelvic inflammation of the minor or deep infiltrating endometriosis (DIE) of the rectovaginal septum (posterior recess). In these clinical situations, the preparation in both compartments should be performed acutely, avoiding blunt tissue delamination and following the principle of preparation as close to the uterus as possible. This method of preparation significantly reduces the risk of cystotomy or colorectal damage. 4. Intraoperative damage to the smaller pelvic organs (intestines, bladder).

If an unintentional cystotomy occurs, it should be used, through palpation, to determine the optimal entrance to the anterior cavity. The hysterectomy should then be completed and the cystotomy should be closed, after prior control (transvaginal or cystoscope) of the location of the ureter and bladder triangle discharges. Cystotomy is performed according to the rules of vesicovaginal fistulas surgery by inserting two layers of absorbable sutures with suture $3-0$. The catheter in the bladder is removed on day 7-10 after surgery.

If the large intestine (rectum, sigmoid) is damaged, it should also be equipped with two layers of single sutures (3-0) running parallel to the axis of the intestine - this does not lead to postoperative stenosis at the site of postoperative scarring. If the bowel injury is less than $50 \%$ of the circumference, no stoma decompression is required.

Occasionally, extremely rare small bowel lesions can also be operated transvaginally after a previous, symmetrical to the site of the lesion, mobilization of at least $10-15 \mathrm{~cm}$ of the bowel loop.

\section{Technique of the laparoscopic excision of the uterus (LH)}

The key to success in the performance of laparoscopic hysterectomies is to perform standard surgical steps in succession.

If the surgeon follows the rules for laparoscopic surgery, the risk of complications is extremely low. The placement of the patient in the correct position and on the appropriate anti-slip mats, the use of the correct instrumentation and the positioning of the trocar are the most important factors. The standard set for TLH should include at least four trocars, two graspers, a laparoscopic dissector, scissors, a medical suction device, a monopolar L-hook electrode, a uterine manipulator, a needle holder and a bipolar instrument. Bipolar instruments with deep vessel coagulation are preferred because they provide a permanent vessel closure of up to $7 \mathrm{~mm}$ diameter. After the correct positioning of the patient on the operating table (as in the vaginal technique), decontamination and dragging of the operating field, and the patient's catherization, the next step is to insert the uterine manipulator. Most of the manipulators available are well accepted, easy to use, reusable and durable. The uterus can be moved in all directions, while the tip of the manipulator expands and stretches the vaginal vault, especially when cutting off the uterus from the vagina with a monopolar L-hook electrode. The vast majority of the manipulators are equipped with a ceramic cap, which creates a flat surface that adheres to the vagina and makes it easier to dissect the bladder even after Caesarean section.

After the uterus is clamped with the manipulator, pneumoperitoneum should be produced. Veress needle technique: to insert the Veress needle, the operating table must be in a horizontal position. The Trendelenburg tilt is carried out after the formation of the peritoneal emphysema and the placement of the optical trocar. The most common entry point for the Veress needle is the umbilical cord plate, because the layers of the abdominal wall are thinnest at this 
level. Before insertion of the needle, determine the course of the aorta via palpation and identify the bifurcation of the hip vessels [21].

The Veress needle must be checked for valve elasticity and gas flow between 6 and $8 \mathrm{mmHg}$ before use. The needle insertion should be at a $45^{\circ}$ angle towards the uterus, with the least risk of damaging large vessels. The abdominal wall should be raised before insertion. In obese patients the insertion angle is close to $90^{\circ}$, while in slim patients the angle is close to $45^{\circ}$. If the first attempt fails, a second attempt should be made before choosing an alternative entry route. Optimally, a pressure of approximately $16 \mathrm{~mm} \mathrm{Hg}$ should be achieved before starting to insert the optical trocar.

No entry technique is completely free from the risk of gas blockage or damage to vessels, intestines or urinary tract. The Palmer point is the safest laparoscopic entry point, as adhesions are the least likely to occur there. For all patients with a much higher risk of adhesions, after abdominal surgery, including Caesarean section, large uterus, umbilical hernias, large ovarian cysts, or a failed bellybutton entry, Palmer described in 1974 an entry point into the abdomen in the medial collarbone line, about $3 \mathrm{~cm}$ below the rib margin. If adhesions are suspected in the left subcostal region, the Lee Huang point should be used, which is located in the midline above the navel and is an alternative to a safe entrance to the peritoneal cavity (Fig. 2) [22].

The next stage is trocar positioning: in the case of a large uterus or high uterine mobility, the optical trocar should be

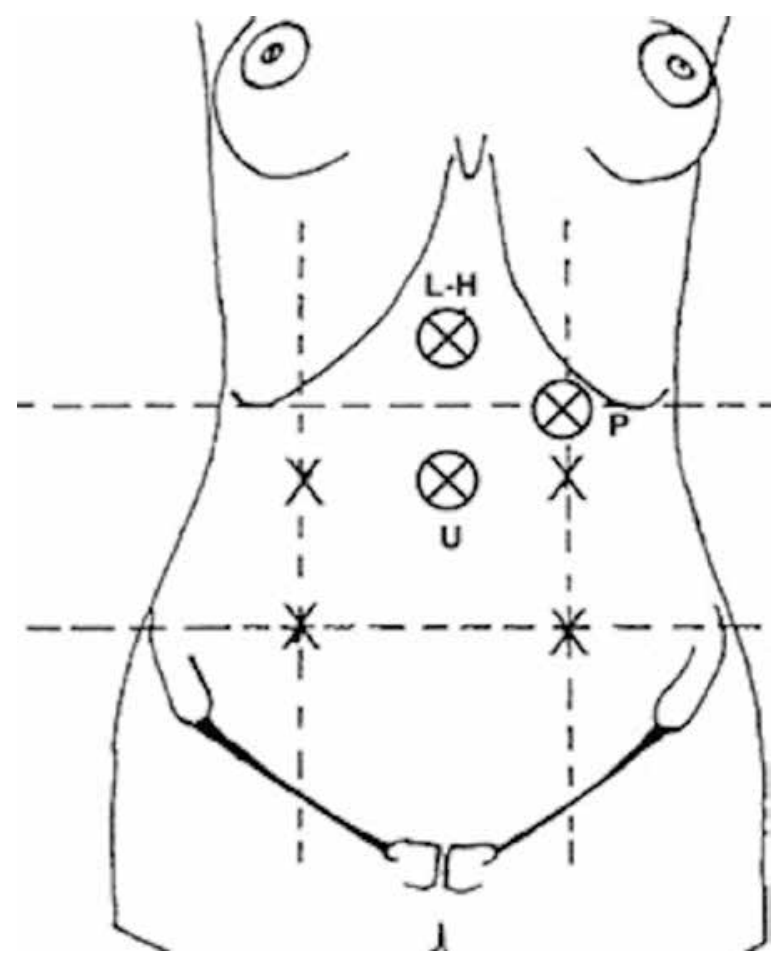

Figure 2. Peritoneal entry points and trocar positioning placed above the navel so that the visualization of the operating field is sufficient. For this purpose, the patient should be re-examined after anaesthesia and then the position of the optical trocar should be determined. Auxiliary trocar should be placed after the manipulator has been put on so that the lower trocar is at the height of the uterine bottom after its removal with the manipulator cranially and the additional trocar should be placed either in the middle line above the joint or laterally at a distance of about $7 \mathrm{~cm}$ from the lower trocar on the operator's side.

After the placement of the vision track and working trocars and the insertion of the uterine manipulator, the entire abdominal cavity, uterine mobility, possible pathologies in the area of appendages, adhesions which, despite appearances, even in the epigastrium may impede the operation from running smoothly, should be assessed before the proper procedure. Only after obtaining the optimal working position and proper pressure - usually about $8 \mathrm{~mm} \mathrm{Hg}$ - can the proper operation begin.

We move the uterus cranially and to the first assistant's side, the assistant grabs the suspensory ligament on the left side by the grasper, the operator cuts the peritoneum showing the retroperitoneal space, and the left ureter. Preparing along the ureter, the uterine vessels are visible. This is helped by positive gas pressure, which penetrates the loose connective tissue and shows the avascular spaces. At this stage it is already possible, although not necessary, for the use of electrocoagulation on the uterine artery at the site of bifurcation.

After separation of the ovarian vessels and incision of the peritoneum of the posterior broad ligament of uterus plate in order to isolate the ureter from possible thermal effects resulting from electrocoagulation, we coagulate the ovarian vessels and cut, then, after coagulation and cutting of the round ligament, the bladder can be separated from the vagina in a safe way, dissecting vesico-uteirne pounch. The uterine manipulator helps us to do this properly tensioned and directed head and towards the bladder. We try to slide the bladder about $1-2 \mathrm{~cm}$ so that there is space to put stitches on the vagina. Please note that the vagina is stretched by the manipulator. After it is removed, the vagina shrinks and the margin necessary for proper suture insertion will be correct only when the bladder is dissected.

After lifting the uterus upwards and cranially and to the right side you can safely remove the peritoneum of the back plate of the broad uterine ligament. This manoeuvre causes the ureter to move away and to show the posterior edge of the vagina and the uterine cut-off.

At this stage the uterine vessels on the left side are already visible, which are coagulated and cut above the edge of the collar of the uterine manipulator, so that if additional coagulation is needed a safe distance from the ureter is maintained. 
We proceed in the same way on the opposite side, moving the uterus further upwards, this time to the left side.

We cut the uterus from the vagina at the height of the upper edge of the manipulator, starting from the right side at the height of the cut right uterine vessels. Then we go backwards in circles and finally move to the front vaginal wall. This technique allows you to easily identify the vaginal edge. By cutting the back of the vaginal wall first, we do not cause the uterus to fall, and thus it is easier for us to see the uncut front vaginal edge of the vagina and safely cut the uterus from the vagina in the next stage. We remove the uterus and the manipulator through the vagina, then seal the vagina to allow the vagina to suture.

There are many techniques for closing the vaginal stump. Many people use Z-type anchor stitches for vaginal angles and then either continue with single or continuous stitches. The authors prefer a 2-0 continuous self-anchored seam. It is important to always check that the vaginal mucous membrane has been sewn during closing. Difficulties in the continuous stitching are most often found in the opposite corner. If the vagina is not well prepared then you should think about putting on a single seam, it will be easier and safer.

After the removal of the uterus and suturing of the vagina, we always carry out an inspection of the abdominal cavity with particular emphasis on the ovary and uterine vessels. First, we remove the working trocars, evacuate the excess gas and remove the optical trocars under a laparoscopic control.

\section{Additional notes:}

Intraoperative damage to the smaller pelvic organs (intestines, bladder).

The most common complication of a laparoscopic hysterectomy is damage to the urinary system. Bladder damage occurs most often during the preparation of the anterior wall and cervix, especially in patients after previous Caesarean section. The cystotomy is delivered laparoscopically with two layers of soluble sutures $3-0$, then we check the tightness of the suture using a cystoscope. The Foley's catheter is left in the urinary bladder to a minimum of 7 days after the procedure. A very important element of laparoscopic uterine removal is to visualize the course of the ureter, which reduces the risk of its damage. Depending on the operator's experience, the ureter can be supplied laparoscopically with a cystoscopy and a probe inserted into the ureter. Whenever after $\mathrm{LH}$ we are not sure of the continuity of the urinary tract, we should use a cystoscope. It is not necessary to do this routinely after each laparoscopic hysterectomy [23, 24].

Bowel damage is a less common complication. Depending on the location of the damage, its extent and the experience of the operator, it can be delivered by laparoscopy or laparotomy [25].

\section{SUMMARY}

Hysterectomies are one of the most common gynaecological operations. We know different ways to perform them, including minimally invasive methods, which are not only safer but also economically more beneficial than laparotomy. An appropriate look at the selection of the surgical method gives the possibility to reduce the number of hysterectomies for non-oncological reasons from transabdominal access. Training of personnel in transvaginal and laparoscopic techniques should be extensive among obstetrics and gynaecology adepts so that the selection of the least invasive method of uterine removal is not limited by lack of skills.

\section{REFERENCES}

1. Committee Opinion No. 701. The route of hysterectomy for benign disease. American College of Obstetricians and Gynecologists. Obstet Gynecol. 2017; 129: 155-159.

2. Miskry T, Magos A. A national survey of senior trainees surgical experience in hysterectomy and attitudes to the place of vaginal hysterectomy. BJOG. 2004; 111(8): 877-879, doi: 10.1111/j.1471-0528.2004.00204.x, indexed in Pubmed: 15270942.

3. Aarts J, Nieboer T, Johnson N, et al. Surgical approach to hysterectomy for benign gynaecological disease. Cochrane Database of Systematic Reviews. 2015, doi: 10.1002/14651858.cd003677.pub5.

4. Lefebvre G, Allaire C, Jeffrey J, et al. SOGC clinical guidelines. Hysterectomy; Clinical Practice Gynaecology Committee and Executive Committee and Council, Society of Obstetricians and Gynaecologists of Canada. J Obstet Gynaecol Can. 2002; 24(1): 37-61.

5. Garry R. The future of hysterectomy. BJOG: An International Journal of Obstetrics and Gynaecology. 2005; 112(2), doi: 10.1111/j.1471-052 8.2004.00431.x.

6. Reich H. Laparoscopic oophorectomy and salpingo-oophorectomy in the treatment of benign tubo-ovarian disease. Int J Fertil. 1987; 32(3): 233-236, indexed in Pubmed: 2885289.

7. Mettler L, Semm K, Lehmann-Willenbrock L, et al. Comparative evaluation of classical intrafascial-supracervical hysterectomy (CISH) with transuterine mucosal resection as performed by pelviscopy and laparotomy--our first 200 cases. Surg Endosc. 1995; 9(4): 418-423, doi: 10.1007/BF00187164, indexed in Pubmed: 7660267.

8. MiskryT, Magos A. Randomized, prospective, double-blind comparison of abdominal and vaginal hysterectomy in women without uterovaginal prolapse. Acta Obstet Gynecol Scand. 2003; 82(4): 351-358, doi: 10.1034/j.1600-0412.2003.00115.x, indexed in Pubmed: 12716320.

9. Kala E, Stojko R, Sadlocha M. Hysterectomy costs depending on operational technique. Ginekol Pol. 2018; 89(12): 672-676, doi: 10.5603/GP.a2018.0113, indexed in Pubmed: 30618034.

10. S RK. 28 Years of Using Hysterectomy Guidelines to Determine the Feasibility of Vaginal Hysterectomy. Gynecology \& Obstetrics. 2015; 06(04), doi: 10.4172/2161-0932.1000375.

11. Chrysostomou A, Djokovic D, EdridgeW, et al. Evidence-based guidelines for vaginal hysterectomy of the International Society for Gynecologic Endoscopy (ISGE). Eur J Obstet Gynecol Reprod Biol. 2018; 231: 262-267, doi: 10.1016/j.ejogrb.2018.10.058, indexed in Pubmed: 30447552.

12. ACOG Committee Opinion No. 444: choosing the route of hysterectomy for benign disease. Obstet Gynecol. 2009; 114(5): 1156-1158, doi: 10.1097/AOG.0b013e3181c33c72, indexed in Pubmed: 20168127.

13. Cho HY, Park ST, Kim HB, et al. Surgical outcome and cost comparison between total vaginal hysterectomy and laparoscopic hysterectomy for uteri weighing > 500 g. J Minim Invasive Gynecol. 2014; 21(1): 115-119, doi: 10.1016/j.jmig.2013.07.013, indexed in Pubmed: 23932973.

14. Wright JD, Herzog TJ, Tsui J, et al. Nationwide trends in the performance of inpatient hysterectomy in the United States. Obstet Gynecol. 2013; 122(2 Pt 1): 233-241, doi: 10.1097/AOG.0b013e318299a6cf, indexed in Pubmed: 23969789.

15. Zimmer M, Pomorski M, Kamiński P, et al. Polish Society of Gynecologists and Obstetricians Guidelines for the application of hysteros- 
copy in gynecology. Ginekologia Polska. 2019; 90(8): 482-489, doi: 10.5603/gp.2019.0083.

16. Shiota $M$, Kotani $Y$, Umemoto $M$, et al. Indication for laparoscopically assisted vaginal hysterectomy. JSLS. 2011; 15(3): 343-345, doi: 10.4293 /108680811X13125733357151, indexed in Pubmed: 21985721.

17. Pölcher M, Hauptmann S, Fotopoulou C, et al. Kommission Ovar of the Gynecologic Oncology Study Group (AGO). Should Fallopian Tubes Be Removed During Hysterectomy Procedures? - A Statement by AGO Ovar. Geburtshilfe Frauenheilkd. 2015; 75(4): 339-341, doi: 10.1055/s-00351545958, indexed in Pubmed: 26028692.

18. Doucette RC, Sharp HT, Alder SC. Challenging generally accepted contraindications to vaginal hysterectomy. Am J Obstet Gynecol. 2001; 184(7): 1386-9; discussion 1390, doi: 10.1067/mob.2001.115047, indexed in Pubmed: 11408857.

19. Sirota I, Tomita SA, Dabney $L$, et al. Overcoming barriers to vaginal hysterectomy: An analysis of perioperative outcomes. J Turk Ger Gynecol Assoc. 2019; 20(1): 8-14, doi: 10.4274/jtgga.galenos.2018.2018.0021, indexed in Pubmed: 30209028.
20. Tohic ALe, Dhainaut C, Yazbeck C, et al. Hysterectomy for benign uterine pathology among women without previous vaginal delivery. Obstet Gynecol. 2008; 111(4): 829-837, doi: 10.1097/AOG.0b013e3181656a25, indexed in Pubmed: 18378741.

21. Veress J. Neues Instrument zur Ausführung von Brust-und Bauchpunktionen und Pneumothoraxbehandlung. Dtsch Med Wochenschr. 1938;64:1480-1.

22. Mettler L, Semm K, Semm K. Hysterectomy via laparotomy or pelviscopy. A new CASH method without colpotomy. Geburtshilfe Frauenheilkd. 1991; 51(12): 996-1003, doi: 10.1055/s-2008-1026252, indexed in Pubmed: 1838998.

23. Malinowski A, Makowska J, Antosiak B. [Total laparoscopic hysterectomy--indications and complications of 158 patients]. Ginekol Pol. 2013; 84(4): 252-257, doi: 10.17772/gp/1572, indexed in Pubmed: 23700856

24. O'Hanlan KA, Dibble SL, Garnier AC, et al. Total laparoscopic hysterectomy: technique and complications of 830 cases. JSLS. 2007; 11(1): 45-53, indexed in Pubmed: 17651556.

25. Laparoscopic Surgery. Management of Laparoscopic Surgical Complications. 2013: 1-7, doi: 10.3109/9780203026205-2. 